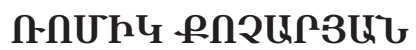

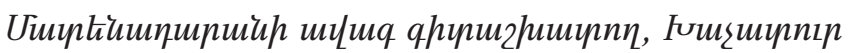

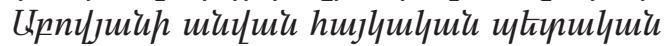

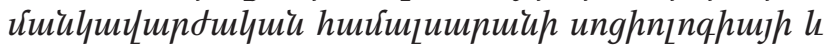

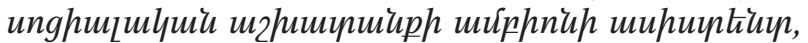

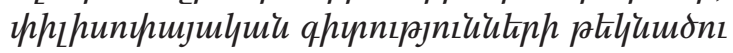

UUU<

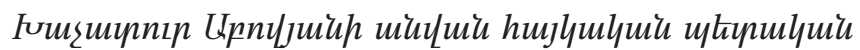

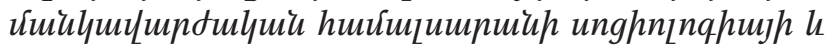

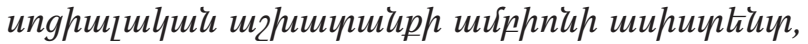

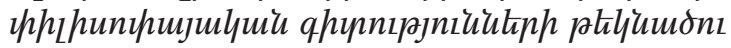

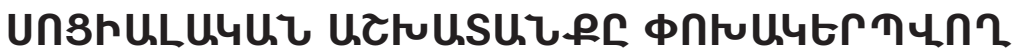

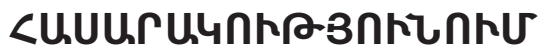

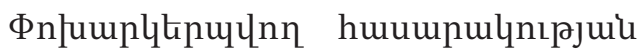

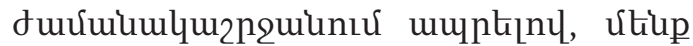

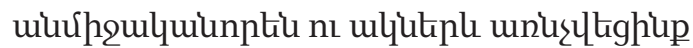

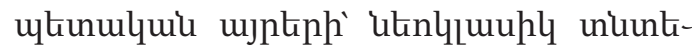

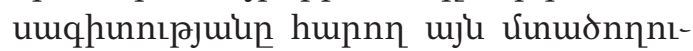

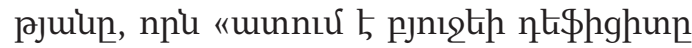

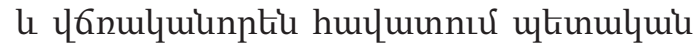
duluuknp uluqkigunn únunkgưuip" [8, 52 10]: Uju ununtignıúu nınпulhhnptiu hpuqnnдцnıर 5 uwl unghu[ulquiu punupu-

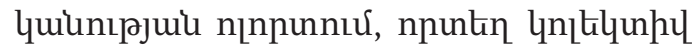

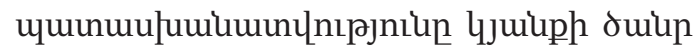

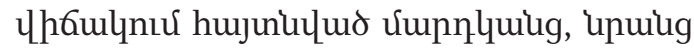
puptilkgnıрjuil le unghuцmlquil uщu-

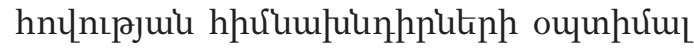

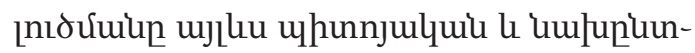
ntilh sh hưưulnuर, tipp huuwpulnnpjuil unghu[ulquil quphputiph huर्ump

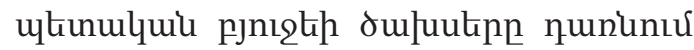

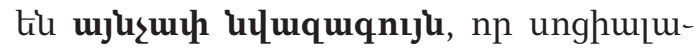

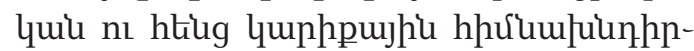
utiph gnıonıún ulqpniupnptiu nununuর wuhumphi: Ujn qnpopupugn huunnıl 5

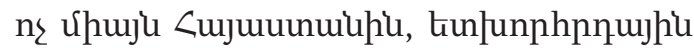

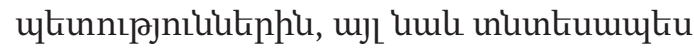

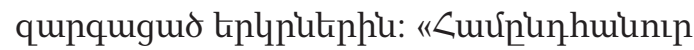

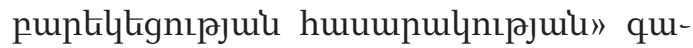

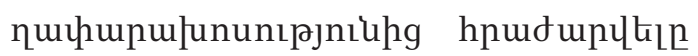
huiqqignkg «uuwuunktph» huuwnulyntpjuiu unknoúuiun [8, 52 10]:

«Unghu[ulyui w2lumunuip» lu "unghu[ulquiu u2łumunnn" huuunhunnınh qujugर्umiu um

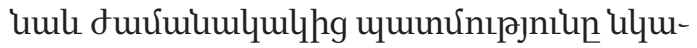
unti 5 unulhu, np hunig h ltip, hul uqqujhu ukinnıpjnitukiph unuqugưứn,

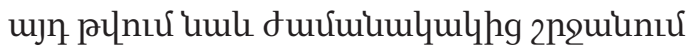

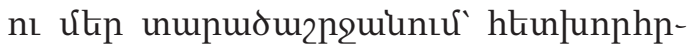

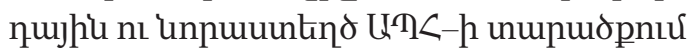

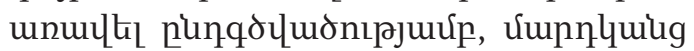
hưưunkn ljuiupnux ulqpniupujhu hp

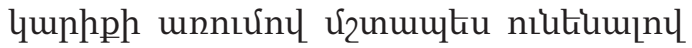

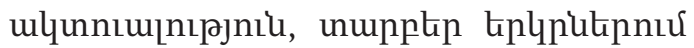
uju huuunhunnıunu hp ulunnıuцnıрjui

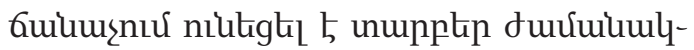

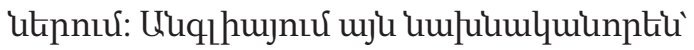

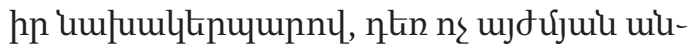

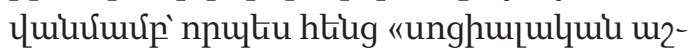

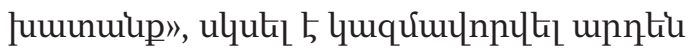

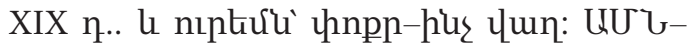

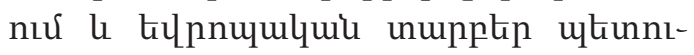

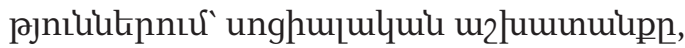
npuku unuilăhu ưuumuqhunulquiu qnp-

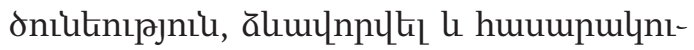


pjuil Utig umulumqhunnıjniultiph guiu-

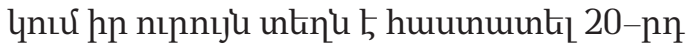
пuph ulqpnuर [6, 52 290-293]: Junphp-

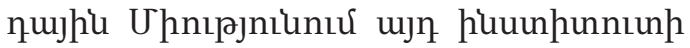

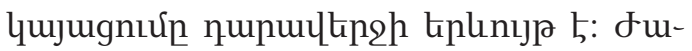

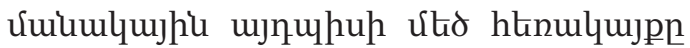

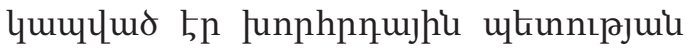
unghuןwlquiu punupulquinıрjuiu htin, tipp «unltiunulquiu punupughi» hurưup-

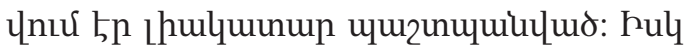
unptil durumumlymlhg hpulquinıрjui

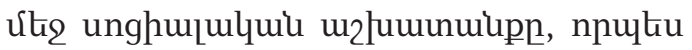

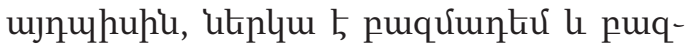

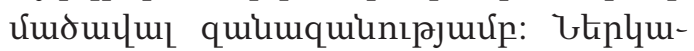

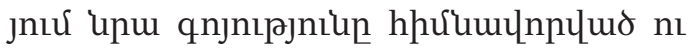

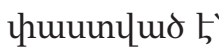

$>$ unnh unghuцwlqui hjuiph uugnt-

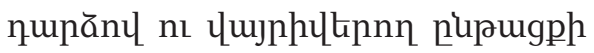

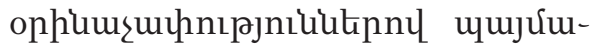

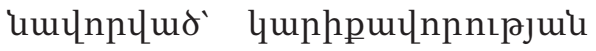
pnik \$tiunútiup lu ujluwje npulen-

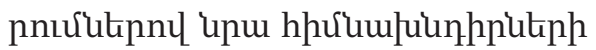

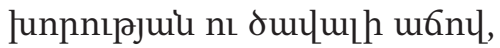

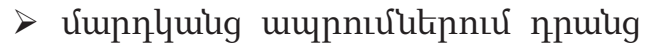

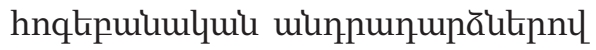

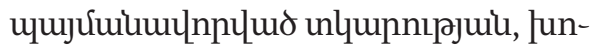

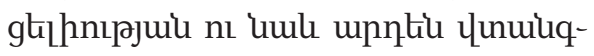

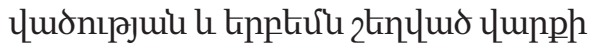
ununuhujunnıpjniuitinnl,

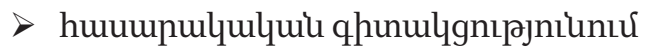

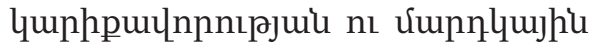
unlumnıpjnıluktph unghwןulquin-

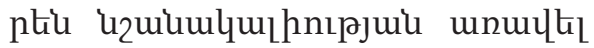
lunp qhunulgưuúp,

$>$ optiuunnulquil nuzinnuर ün दuphpuunnnıрjuil nı unlunnıрjnilutiph ulquinưurip unghųulquil hnquont-

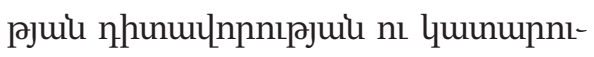
uh uhnnnנulquinıрjui hnuцирu-

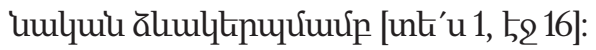

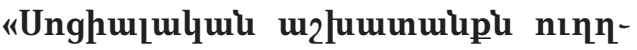

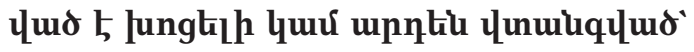
unghu[ulyuil hjuiphg inupuiguin-

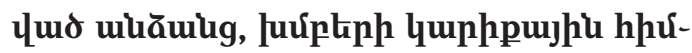

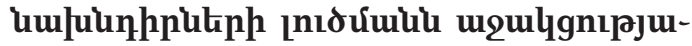
up h huumpulqulywi ljuipntú upwing

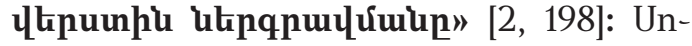

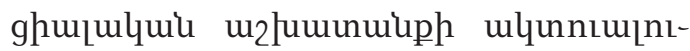

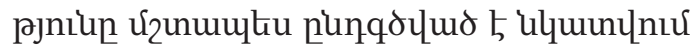
huumpulumph le unghwimumil ljwuph

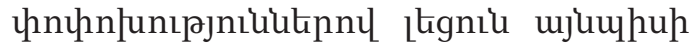
dưưumlqurnquiutinntu, tipp unuцtil

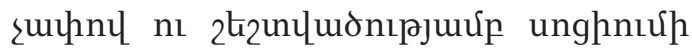

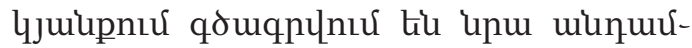

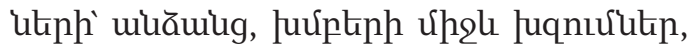
ltiugunuulupnıpjniknuर ns uwl hnqunp

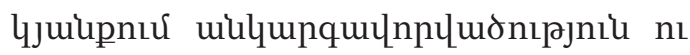

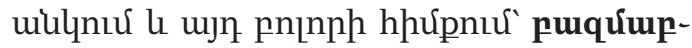

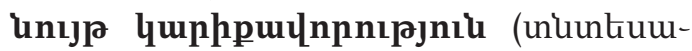

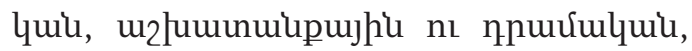
4nрulquil, unnп̨шumuhuluil, ...):

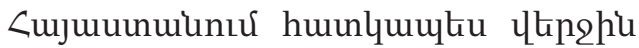
tplns unuukurujulqutinnuर unghu[ulqui u2 luwumuipn pnıni qunqugniu 5 шщ-

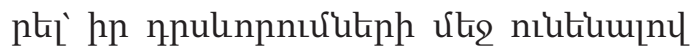
juinppitiph puqúuquiunıpjniu li puq-

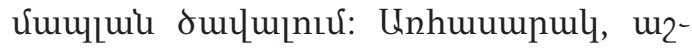

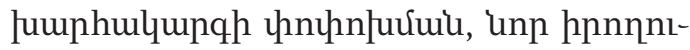

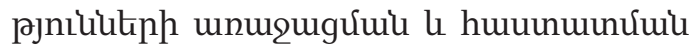

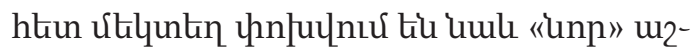

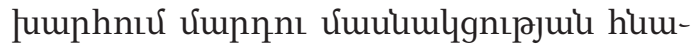

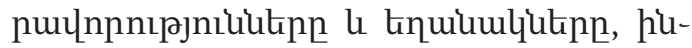

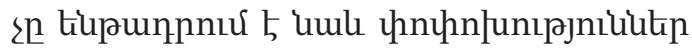
ưpnlquiug uinptiph nцnpunnư puiun!nnunnuर: 9njwzwndn, npu ununuhwjunlti $5 n$

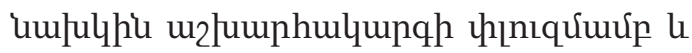
unph unuqugưứ, gugtil $5 p$ umu ưmp-

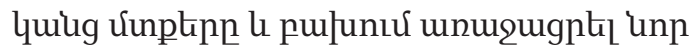

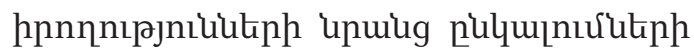

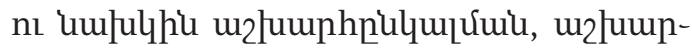

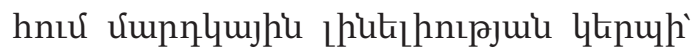
4juiupn, ltiugunuцnnnıрjnıนn quqưu-

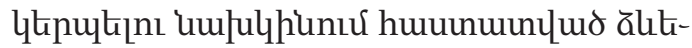
nh úpqu: Unghu्ulquil qnjuzundu wju-

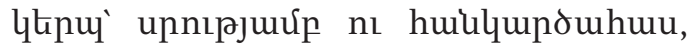
huuupulynıpjuiu ljuiupnıu puguhujunk- 


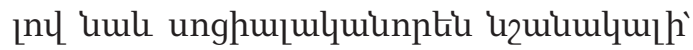

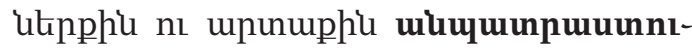

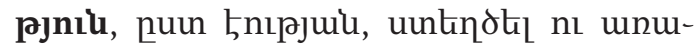

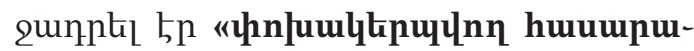

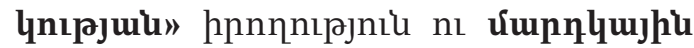

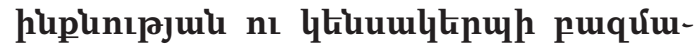
pnuminuly lulnpulyupq:

utupghi 15-20 unuphitiph pupurgpnıu <ujuuunuiunıu unknh nithgnn unghuju-

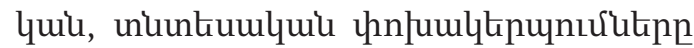

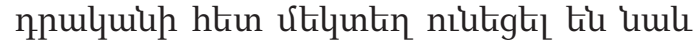
puguuulquil uunpuñupă huumpulynı-

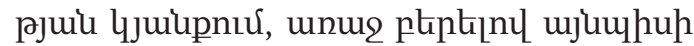

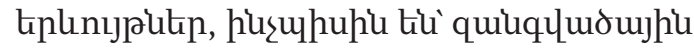
unpuinnıpjniuiu nı qnpouqplynıрjnıนn [4,

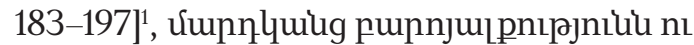
undtpuiunpưujhu huर्umlupqh humplu-

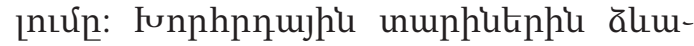
цnplud le qupqugur hiuunhunnıhniuul

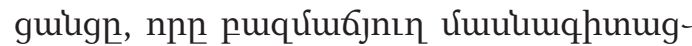

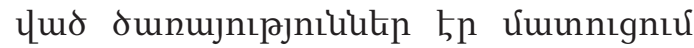

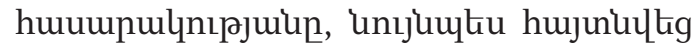

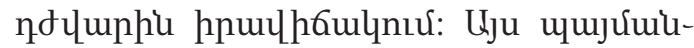

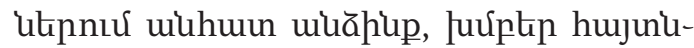
lthghi duin luagnıpjuil uthe: Lpugnighs

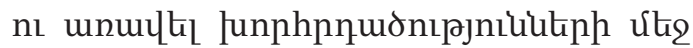
५ unıqniर्u uhunpp uzuiumlyulh uju hpn-

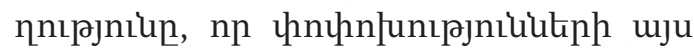

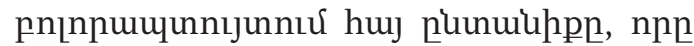
nuptep zupnituul huinhpuugtil 5 huuupulnıрjnín, upu updtpujhi lnnưun-

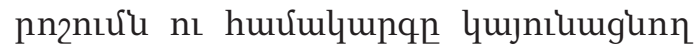
quplinpuqnuju qnponu, unyjuuku hujunilthg Gquudưư út̨:

Uupnluushi punıрjuin ulqpniupn-

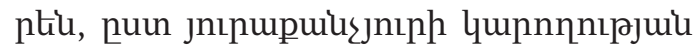
lu qhunulgruiu, huinnıl 5 qupquunn-

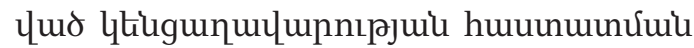

\footnotetext{
${ }^{1}$ Eunjunphpnujhi utiunnıjnitukpnix le huunquultu <ujuuunuiuntu «unp» unpuinnıpjuil $\mathrm{l}$

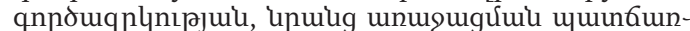

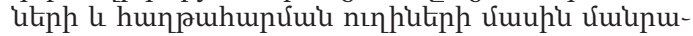

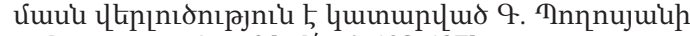
u2łuuunnıpjnilunıu [unt́u 4, 183-197]:
}

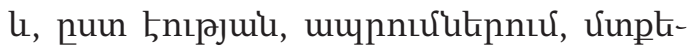

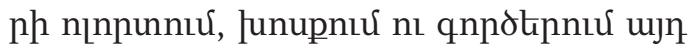

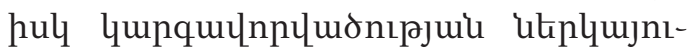

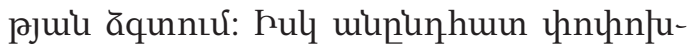

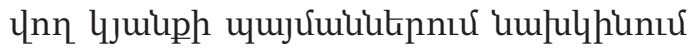

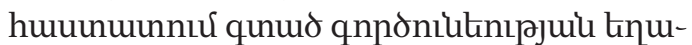

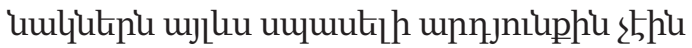
huiuqtigunıu, nununsu bhu wilquilumunt-

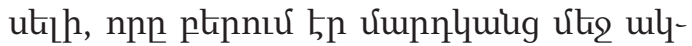

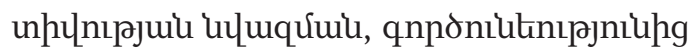

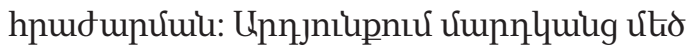

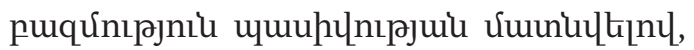

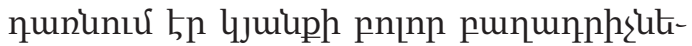
pnıर्u (unghujulyuu, uniunkuulquil, црршquil...), ptul hp umpunhqưujhu unnıर्unl

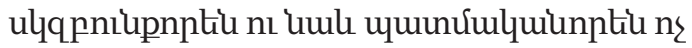
ujupuiu unp, pujg li' uwlulhihg unupptep

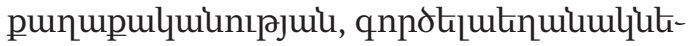
nh, umpph nı pupph inpuhuuunuun hpn-

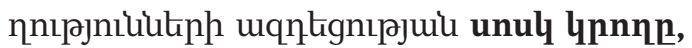

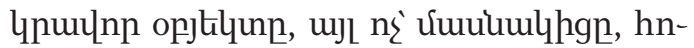

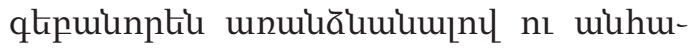

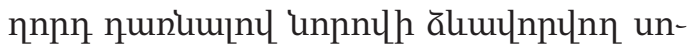
ghuцuluul цjuiphiu:

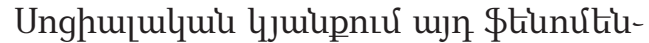
ukph lunpugưuid htinlumupujhì npulenpnứutipn puqưuquiu til, lu hứulu huil-

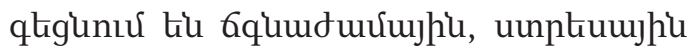

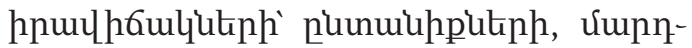

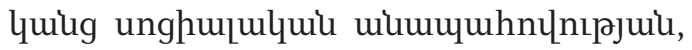

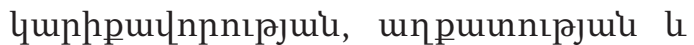

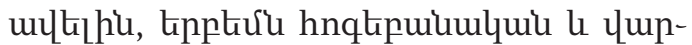

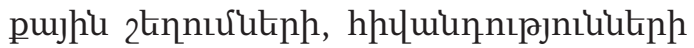
nı unqupnıpjniutukp [5, 5, 107]2: Unghu[u-

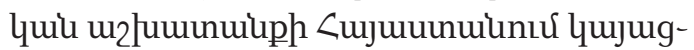
রuil uju puinıpjuil unnıर्un luplinp 5

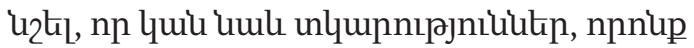
uqujर्umumunplud tiu n々 úpuju unghuju-

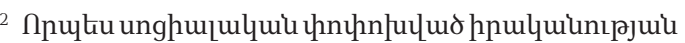

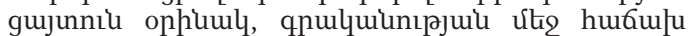

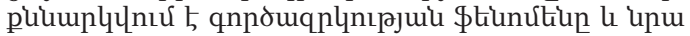

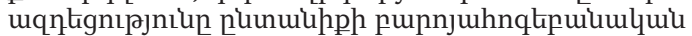

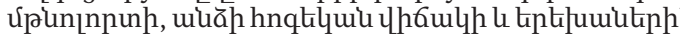

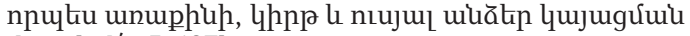
பnu [unḱu 5, 107]:
} 


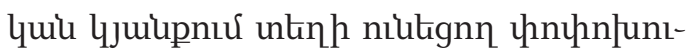

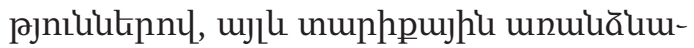

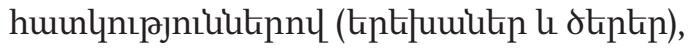

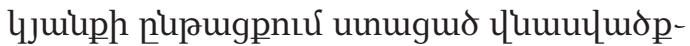

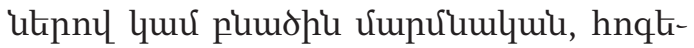

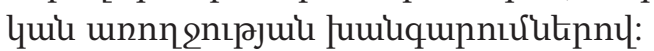

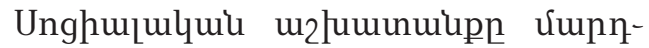

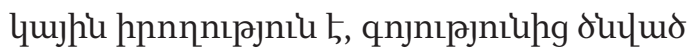

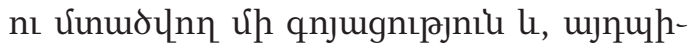

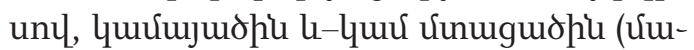

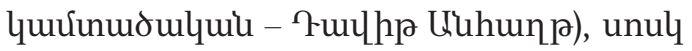

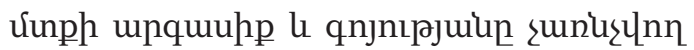

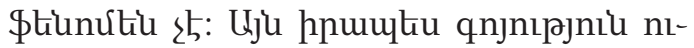

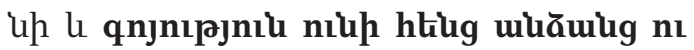

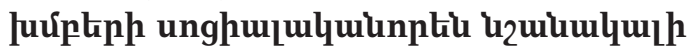
quphpulnpnıрјuil nı unlupnıрјui $\$ \mathrm{t}$ -

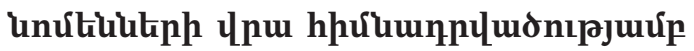

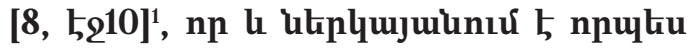
unghu|luluil w2/uwunulph nıunıuuuuppnipjuil l. pnil qnponitinıpjuil

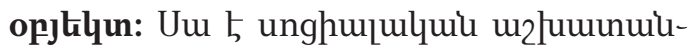

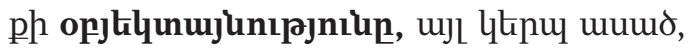

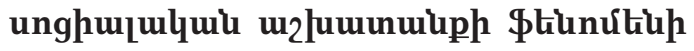
qnjugúul opjtyunujhl hpúluulnplu-

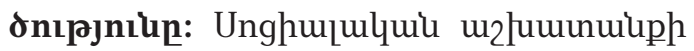

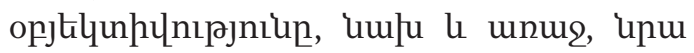
opjtiquwjunıpjnitul 5: Ujuupunl, qupklh 5 uutl, np unghupulquil ljuiph utis

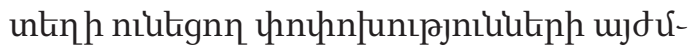

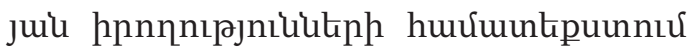
hp unuplqujulquinıpjuर्up (opjtymuj-

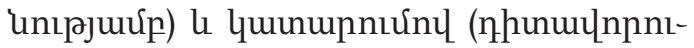

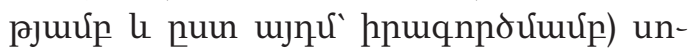

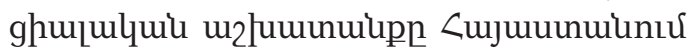

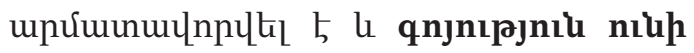
opjtyunpl hpúluunpnıpјứp':

\footnotetext{
${ }^{1}$ Unghujulyuiu w2humunuiph unugugnıún Unpu-

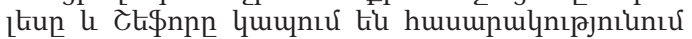

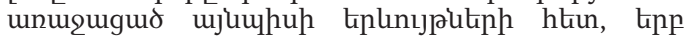

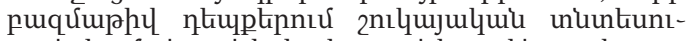

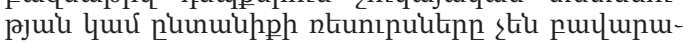

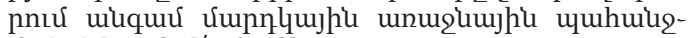
únıluputupn [unḱu 8, 10]:

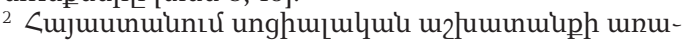

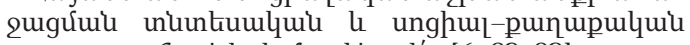

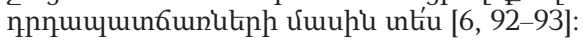

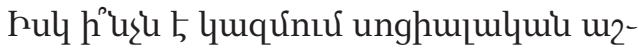

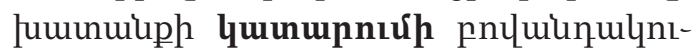

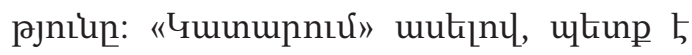
huuluikul unghujulquil u2 umunuiph

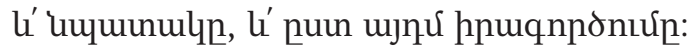
Unghujulquinntiu 'uzwiumlyulh quphpu-

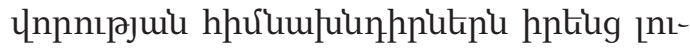
১nıúu tiu qununıu uqulygnıpjulu nınhnu: Glyuuntip, np quphpulnpnıрjuil puqưununıs' hnqunp ns 'ujnıpulquil, thut-

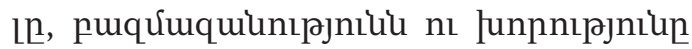
hptiugny unıjuulyuinntiu uujưumuln-

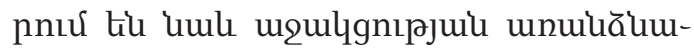
huunlnıрjniuitipn: Ugulgnıpjniup hupl

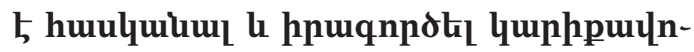

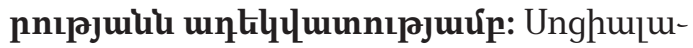

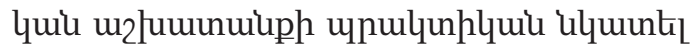
5 unulhu, np <ujuuunuintu ugulggnı-

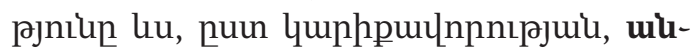
hpudtingnıpjứp ns tuul uupuptunpt-

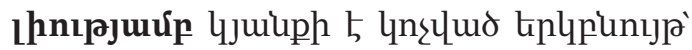
hnqunp nı ujnıpulquil, npulyujhi nı pu-

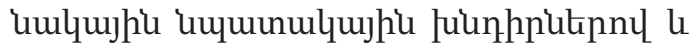

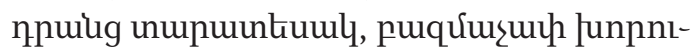

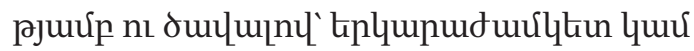

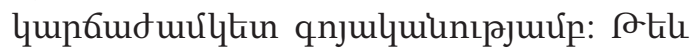

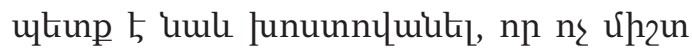

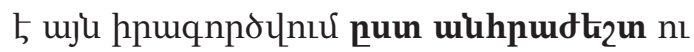

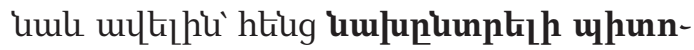
julyuinıpjuil:

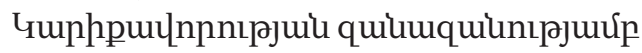

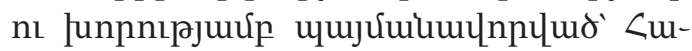
juuunuiuntu unuqughl tiu unghupulyuit 2tpuntep ns lữptep, npnup wiuntuulud ns

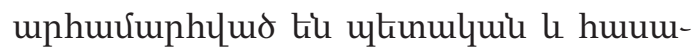

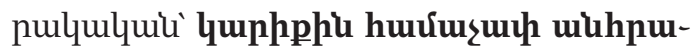

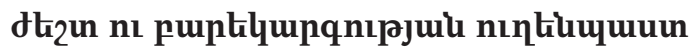

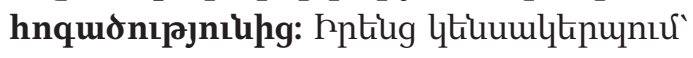

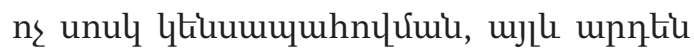

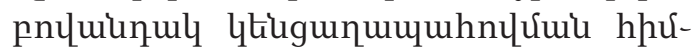

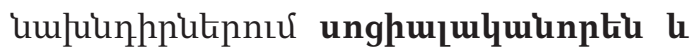
unıjuhul punupulppnptil izuluuquil qupppulnpnipjnil nitugnn un- 


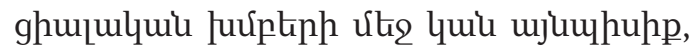

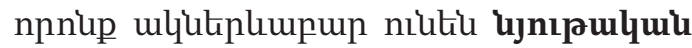
lu hnqunp nı lppulyuil uqulgnıpjuil quphp: Uju unnıunul wihpudti2u nt

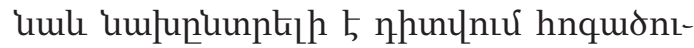

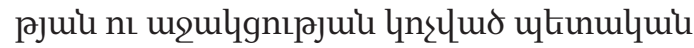

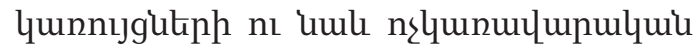

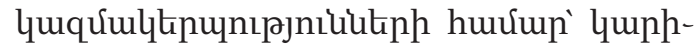

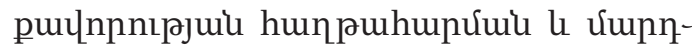
qujhi hupunıрjuiu, puqưupnцmunuly

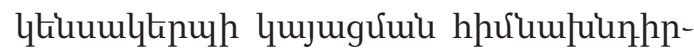

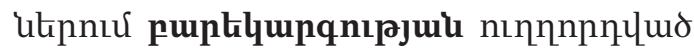

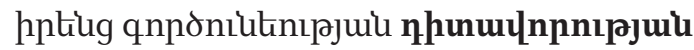

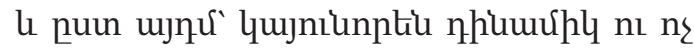

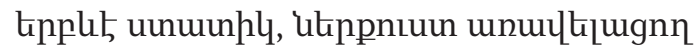

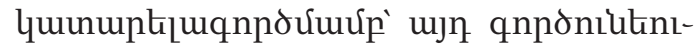
pjuiu hthpumupuinnúp, uju

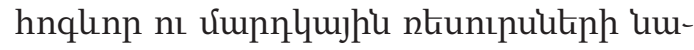

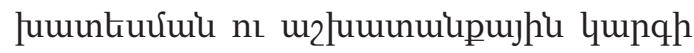

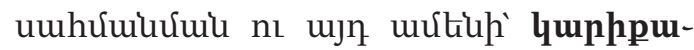
unpnıpjuil hứtiúum umb htinuqu unptilghujh le, ytinguuku, qnnonilutne-

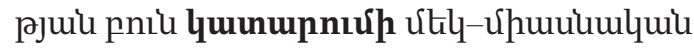

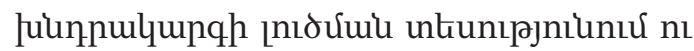
unulunhlumnıर्u ulquinh niltiume puqviuu| ulunıpjnılu:

Unghu[ulquil w2 wumunuiph upul-

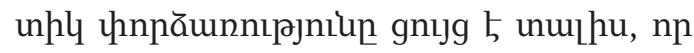

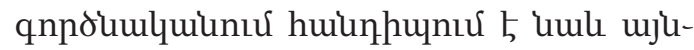

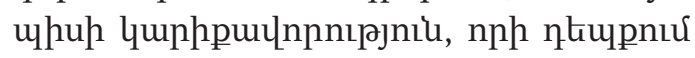

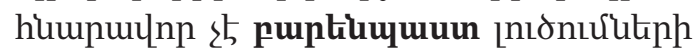

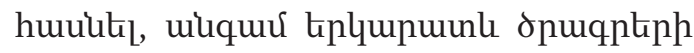

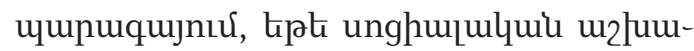

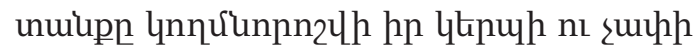

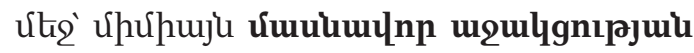
lu ne tipptiứ' quppphi hứmumunum-

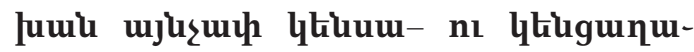
huquuu hnquonıpjuil, tpp unghu[u-

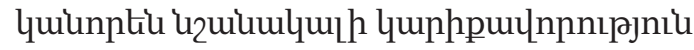

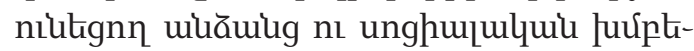

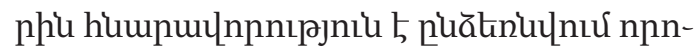

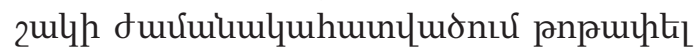
piunuiupp huiumumqonju hugn hnqu-

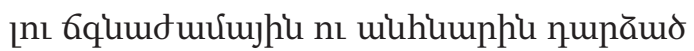
buinnp plinn, ăhnpuquunltal uuqnnnı-

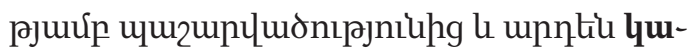

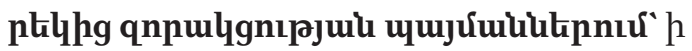
qnnns thutel hpliug uteppiu nteunıpuitepu

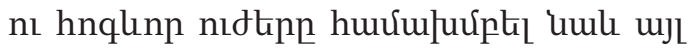

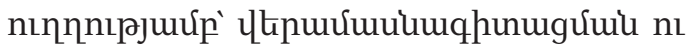
uJl w2/umumuiph, nuunuihpniu unhpnn hnqtipuiumlquil upinnnpunp pupkeuu-

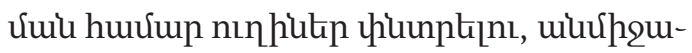

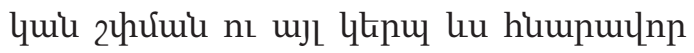

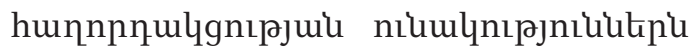
nı 2nquiumlitipi punjujutins, ưnuhn-

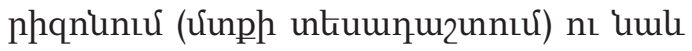

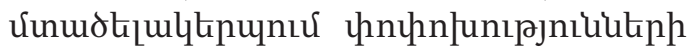
uuhnudti2unnıpjnilup qhunulgtins lu hup-

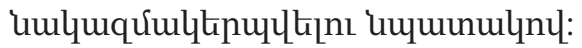

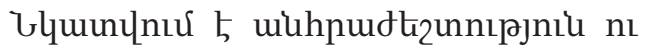

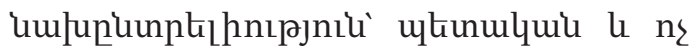
qunuцunulyui qunnıguteph onuqpteph 2nquiumlnnu ulquinh nitituml qupрpu-

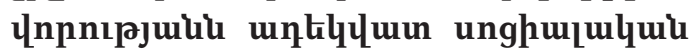

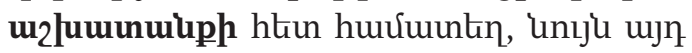
ulqpniuph hưưăuju' hpuqnndti 'ujnı-

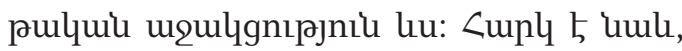
np ujn dpuqptiph ujnıpulquiu lu hnqunp,

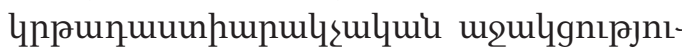
un uumunulyunınпumo lhup wuăming,

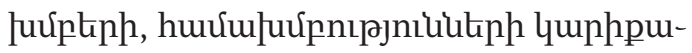

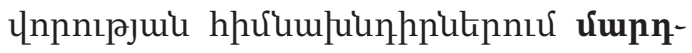
luughu hupunıpjuiu nı huúulgkgnıpjui

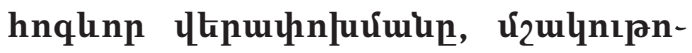
ptil utiplppjul l ujuni punupulppp

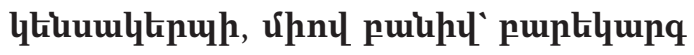
qnjnıрјuil qujugúuin, puiqh цuph-

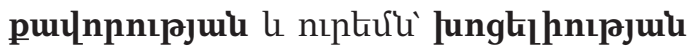
l unıjuhul upntiu lunuiqumonıpjuil

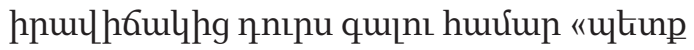

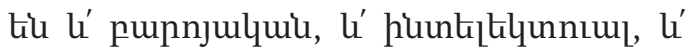

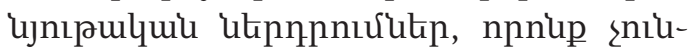

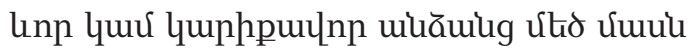
hptiug pnıJL unul stiu qunnn»[3]:

«Unghu[ulqui m2lumunuipp qnjnı- 


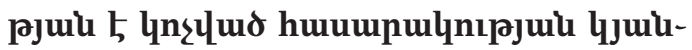
pnıu puptyupqnıpjul huunuunúul quphpny l. nhunulnpnıpjuxíp" [1, 52 20]: Unghuцuluiu ljuupniu punhuipu-

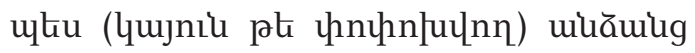

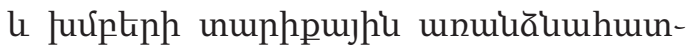

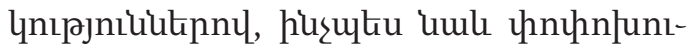

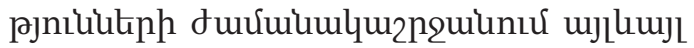
umunfunitipny unugugnn quphpu-

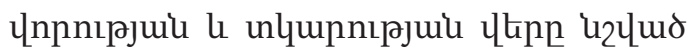
\$tiunútiuknnl uxujumumunplud ungh-

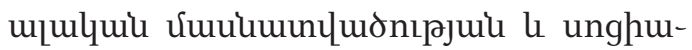

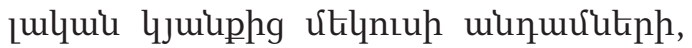

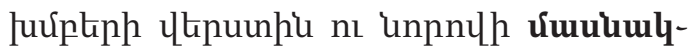

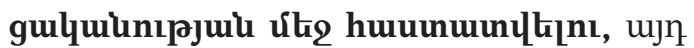
pipurgpniv upuing iluquqnıju wiuhpu-

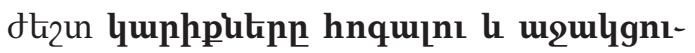
pjuil fuinhpitipn, uju quर्u uju buцu-

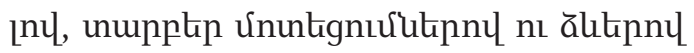

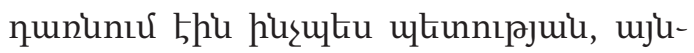

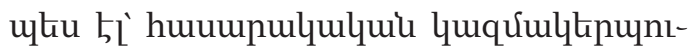
pjnilutiph hnquonıрjuil unupluut: Ujn

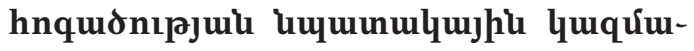

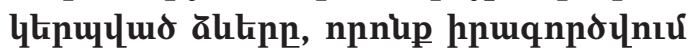
tu wiứpquluil hunnpnulgnıpjứn, hpulyuiumglnıu tiu hting unghujuluul w2luwunuiph úpengnu:

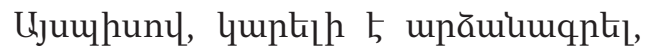
np unghu[ulquil w2 kuwumuiph qnjnıрjnı-

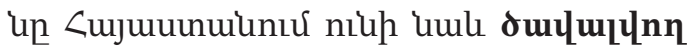
uhunnıর: hǔñ Guphpulnpnepjul puqưuntuul npulinpnıúutipl le huuwpulnıpjuil vitr npuil yuptilyg nı urulyhg umunuuluwi-upăuquipp, lhup qu uthunuyuil yunnıglitpp, pť huuwpulyuluul

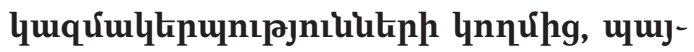

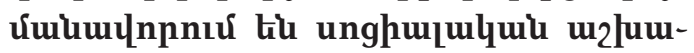

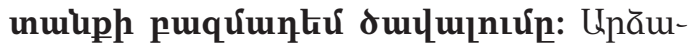
uuqptiup, np unghujuluiu u2łumunump

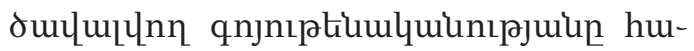
unnil Łnu unupupunıjp puqưuquiunıpjnıtup, पuluцuo nhunulnpnıpjnilhg ('uuuunulhg) lu puunplud opjtyung, unlqu

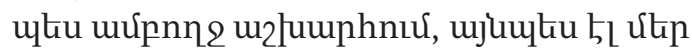

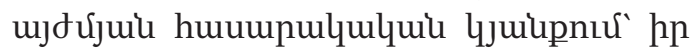
qujugưuil রite purguhujuntinl ns unul

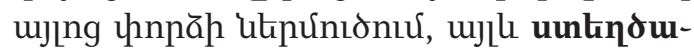
qnpoulyuil utpnıd l. npulınpnıর:

Unghujulquil u2lumunuiph dulu-

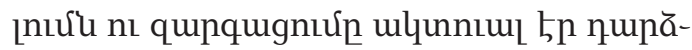

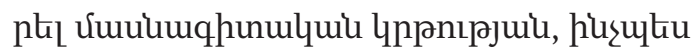

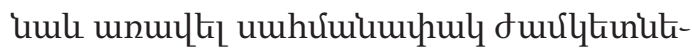

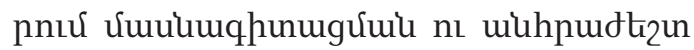

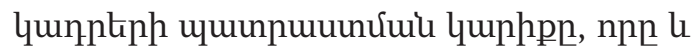
hpulquiuugunıu

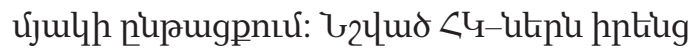

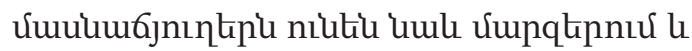

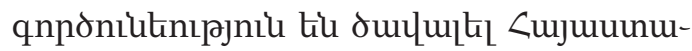

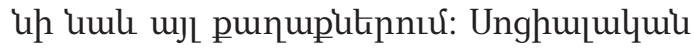

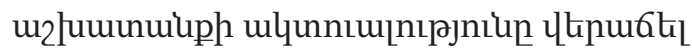
5 viuuluuqhunulyuil yppnıpjuil uihpudtżnnıpjuul, le qupk np n々 úpuju upuntplpniu, wjll huj hpu-

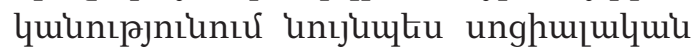
u2 luuunuupu hp npulenpnivíu ns huuunuunuqpnứu

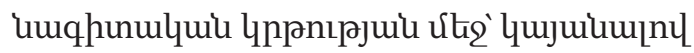
npuktu nıunıgulupq:

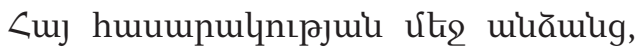

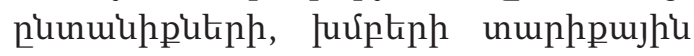

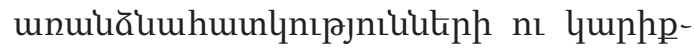
'ukpp l' dulupnl, l' quiumquiunıpjứn

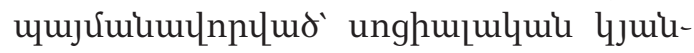
ph uuumuptiqniर uju unuphitiph pipaug-

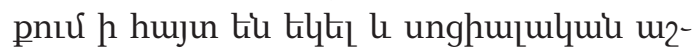

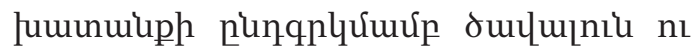
unupupunıjp qnponiutnıpjnilu tiu hnu-

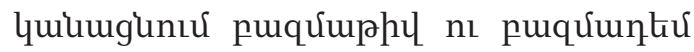

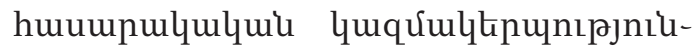
ukp: Ujou qnponıर tiu puquumphl hu-

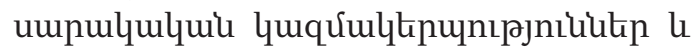

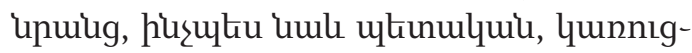

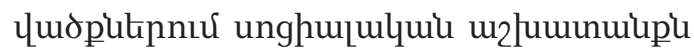
nunqplyum 5 npultu uishudtzin pur- 


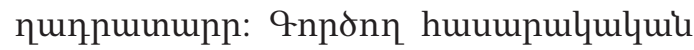

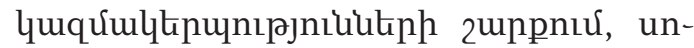

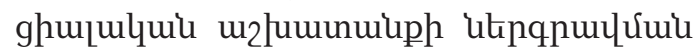

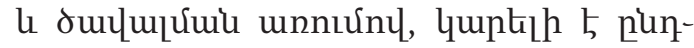
qpltil ne pninp humunulyulumil quq-

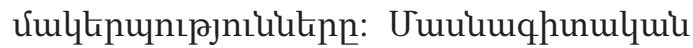

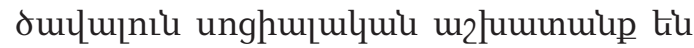
hnulquiumghi «Unuphinıpjnil Cujuuunuiu», "Onnuiu», "Фjniluhl», "<nujuh 4u-

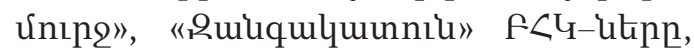
"UnU viulquiluiu qunıntip»-n, MSF-n,

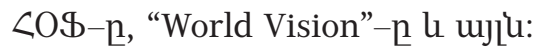

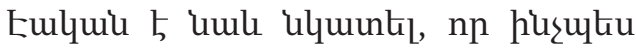

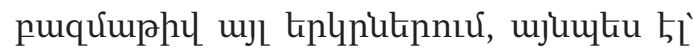

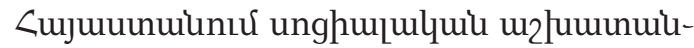
ph unupupunıjp puqưuquinıpjnıutu

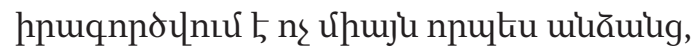

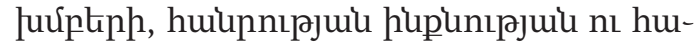
uulykgnıрјui unupuuntuml, ynulphtion

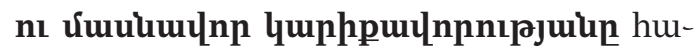

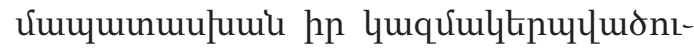

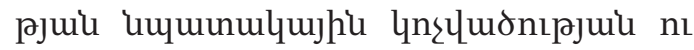
ălekph unwhunplynptiu puqúumbuml $\mathrm{l}$

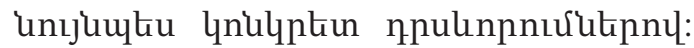
Eplpunus hn upunwhujunnıpjniutinnuर l' hnqunn, l' ujnıрulquil, qunhpuunnnı-

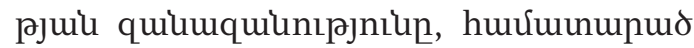
lhukiln le jnıpupuisjnın hn untumlynıu

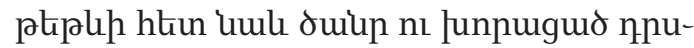

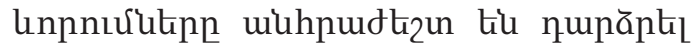

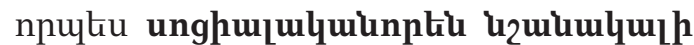

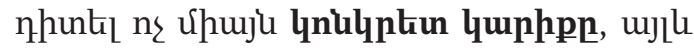
ulqpniupnptiu qupppp nputsu uјnupupl, ujuhlpi punhwinıp quphpp quर्u

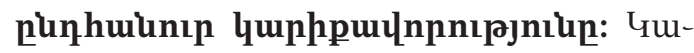

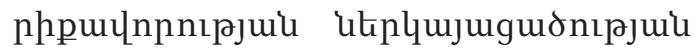
lnulptinnıpjniuhg pugh upptiu uul

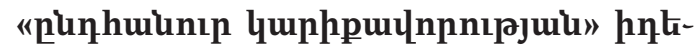

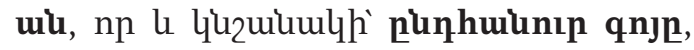
npuku nızuпрnьрјшu, шщu tuwh huulugnn hnquonıрjuil $\mathrm{l}$ щшunuu|umil-mpauquiuph uuhpudtizu ne uphnoulyuiu

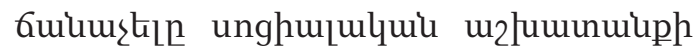

qnjnıptiumquinıpjuin unupupinı

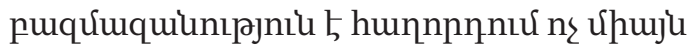
npuku ujuupup \$tiunutiu, npu upnuhujunnuर 5 unul qnponittenıpjul thpu

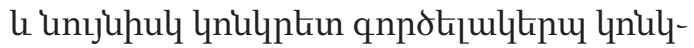

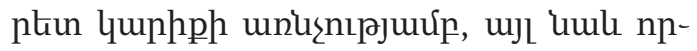
uliu qhunnıpjnitu:

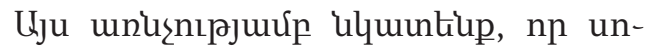

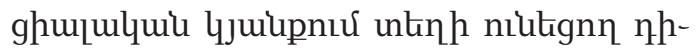

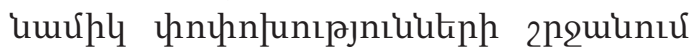
quphpuunnnıрjuiu junpugưuin npulku umunuułumu unămquiup unghmןmlymu

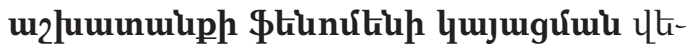

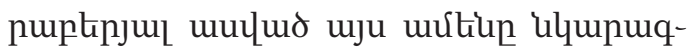

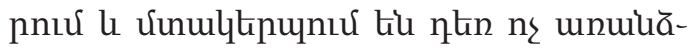

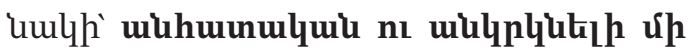
ppnnnıpjnit: Ujuinkn bulqui 5 hh2w-

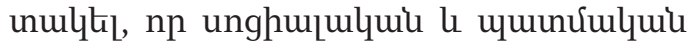
qhunnıpjnilutipnıu, nuin Ч. Ү-һцpujh, npuku hkunuqnunnıрuuiu ulqpnikpujhu

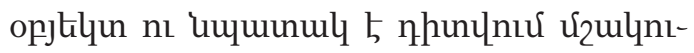
pujjhu nı unghu[ulqui hpnnnıрjnilukph qnjuц|nnnıрjuil únnnıupu punnn2 ulumu-

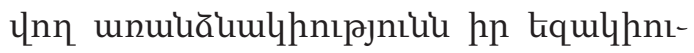

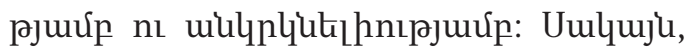
ujl 5 ll vitaq nunniuklh' Ч. Rhıpujh hten-

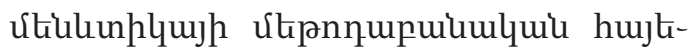
gulyunqhg' sulquinntiu 'uzwiumuulh

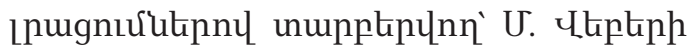

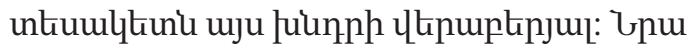
«huulugnnulqui unghn[nqhujh» hujt-

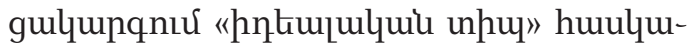
gnıpjuiu úhengny huulqugumiu tiupulqu

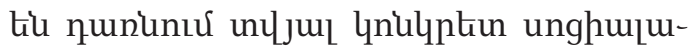
quiu hpnnnipjnilu npulquutiu pntiu-

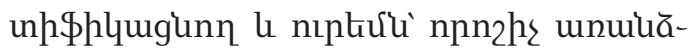
'umhumlnıрjniuutinn' wJ Gnulphtion unghuцuluil hnnnnıpjniukteph htin hp punhuiunnıрjuil hpuqnpдцuðnıрјuर्up no uwal htunuqujnux lu nunhwuinuiumthnıрjui unnutiughujnц: Unıju hрuшш-

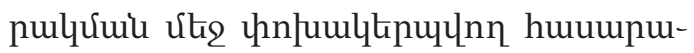

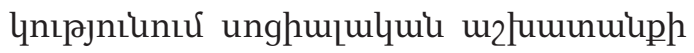




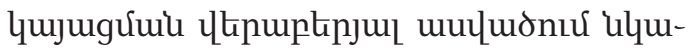

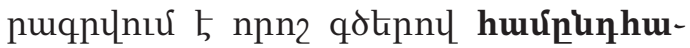
unıp \$tunútu, husn nputue ujnuhupi nı uule npuktu úpunnı nı unghujulyul nhiuuर्ulyu' punpn2 5 kunlunphpqujhì

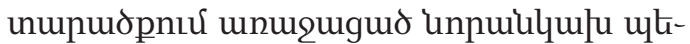

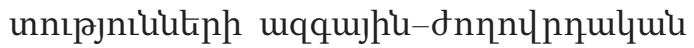

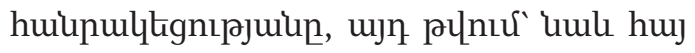
hpulquinıрjuiln:

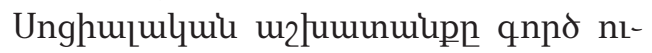
utiumpny quphpulnpnıpjuil l. unlupnı-

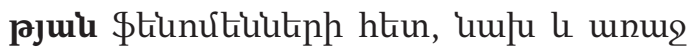
nıunıর'uuuppnıর 5 npuiup, le lthpohiukphu huulqugnıর'u nı purguhujunnıर्u,

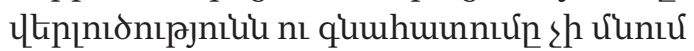

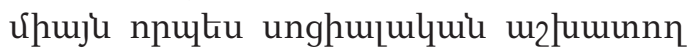

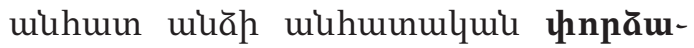

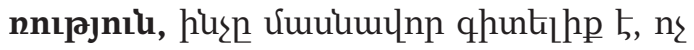
uh2u unfuuigh

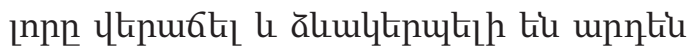
npulku "ninhwuinıp lư plinhuilpulyuu qhuntlhp", hišn 'uzuiuulynıu 5, np ujlu ujuuhup qhunthp 5, nnp qupklh 5 thnluula-

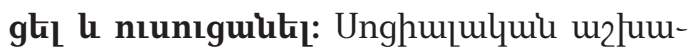
unuipu ujuuhunl unul thnpăunnıpjnì

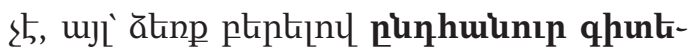
lhph qunqulh6ul' qhunnıpnit 5 l- -quर्u upltuun, nıunıguitilh ujıng, npnl li qujugkl nı qujuinıu 5 npulku qhunu-

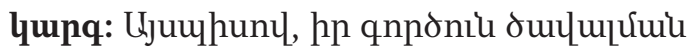
l. huuunulyuiuugnn huuunumuqpnıрjuil qnpopipugniv unghujulquil u2lum-

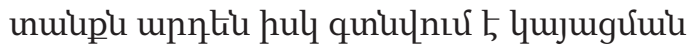

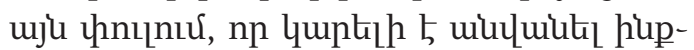

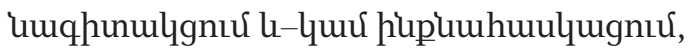

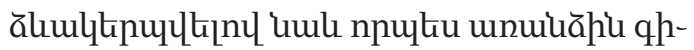
unu6jnın दuu qhunulyunq: Ujuuphup lup-

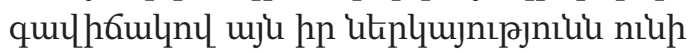
n乞 uhuju upuntplpnıর, ujll huj hpulqu-

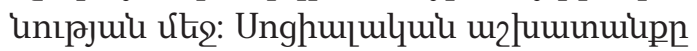

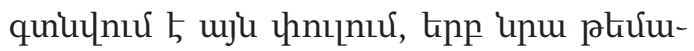
unplujnц qpцnư tiu le lqpцtiu qhunulyuu

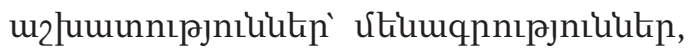

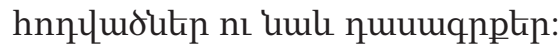

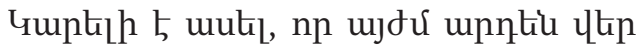

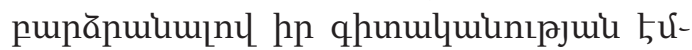
uhphl qupquilh6ulhg, uju hp pn-

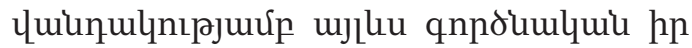

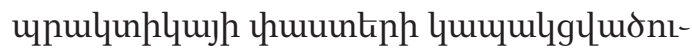

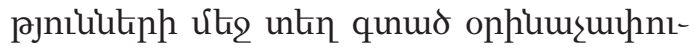
pjnitutiph unul «nhunuplynứutpp hu-

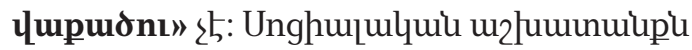

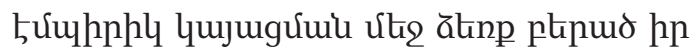

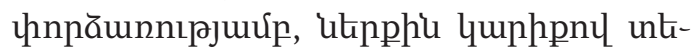

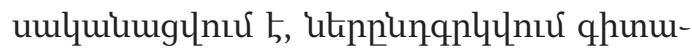

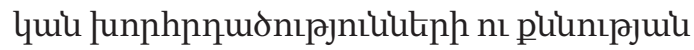

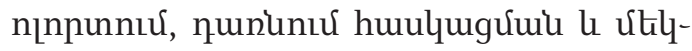

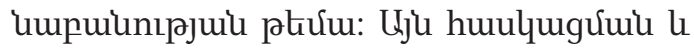

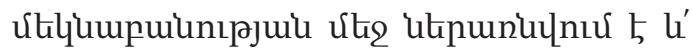

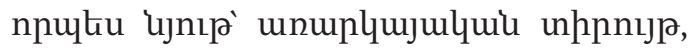
npp htiun qnpo niup qhunulquimptil unt-

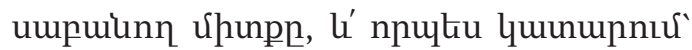

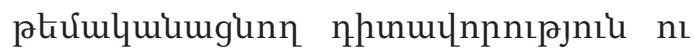

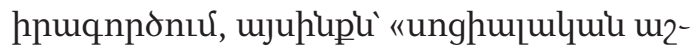

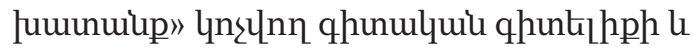
htiug qhunulqunqh qujugnıu:

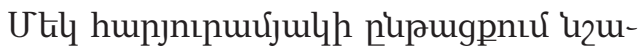

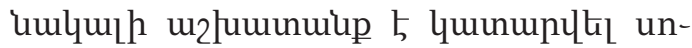

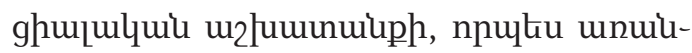
ăhu vuuumqhunuluiu qnponiutnıрjuil,

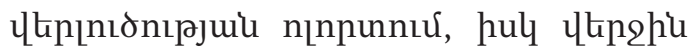

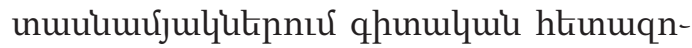
unnıpjnitutpnıর unultal pinqdyudnı-

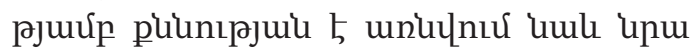
upntiu npultu pupunıpnıju unghuju-

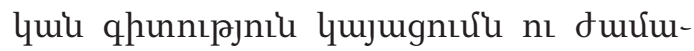

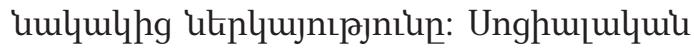

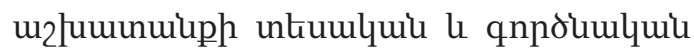
pununphsiknp lkpuptinjui qnjnıpjnil nitugnn qhunulyuiu pulumplynưutenp 2u-

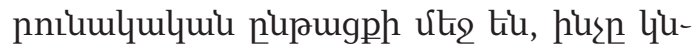
2uivulh uul, np unghujulquil w2 wumunuiupp qunulnıর

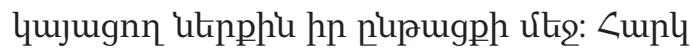

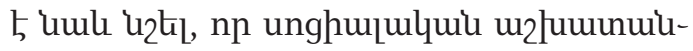

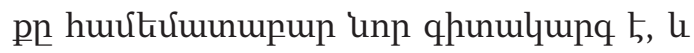




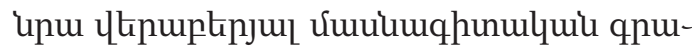

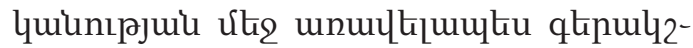

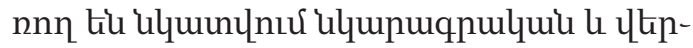

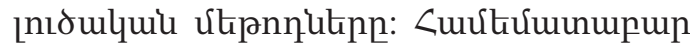
wцth phen huqumntru tiu puiumuunupup ltipןnıonıрjnil hpulquiumgunn

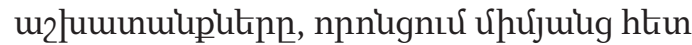

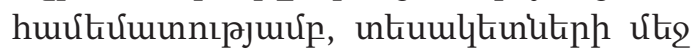
puimulk6h, qunumuphtiph dunuiqq-

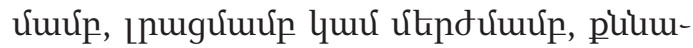

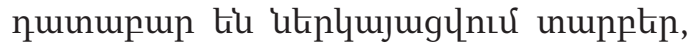

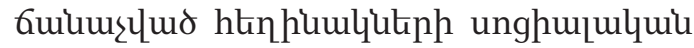
u2luwunuiph nцnpunnư hujunup nunămo

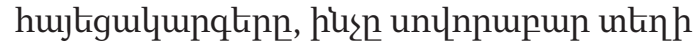
5 niutiunuर qhunulyunqtiph umunúulquiunptiu qujugnn qunqugúuiu pupugpntú npuku qhunnıpjnil hplikg qnjnıpjniup

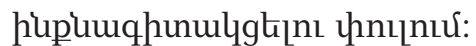

<ujuuunuiunư qnnduninn $<\mathrm{G}$-ukph le ukinulquil qunnıgumophtiph lnnर्uhg

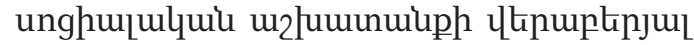

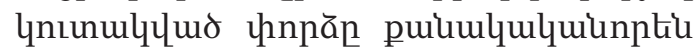

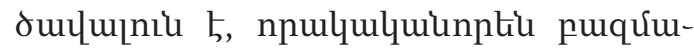
punujp, le upntil ulquintilh 5 ujn thnp-

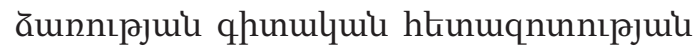

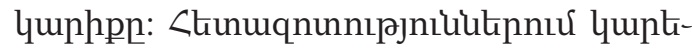

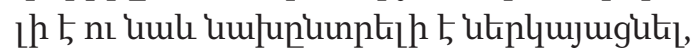

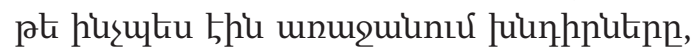

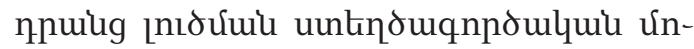
unkgnıưlukn, bứpujhu puiumplnnưutenp, npnug pupugpnư, puunıрjuiu unitinnl

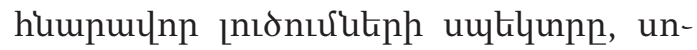

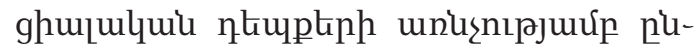

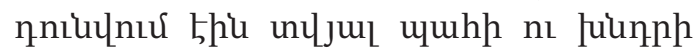

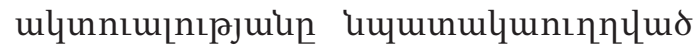

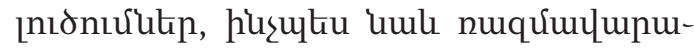

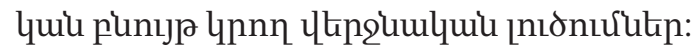

Unghurulquil w2tumumuiph zunniku-

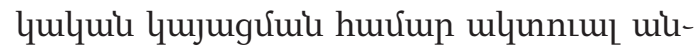

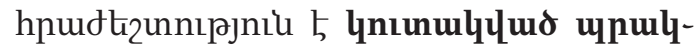
unl tenpăunnıpjuì qpunulquinptì huulqugnıúp, úzulynıúp, Uthliupuinnpjnilup $\mathbf{l}$, ujuupunl, npute upntil punhwupulquil nunlume qhuntelpp nt- unıguitilh l nıunıgulump, 中n|umil-

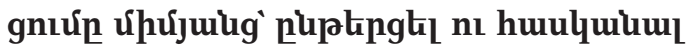
aqunn unuiph «huphumqhumulgưulu» le npulu qhunulqunq zwpnitumlqulqui qujugưui nı qunqugưuiu pnınn thnцlinnıर्u Eulqui

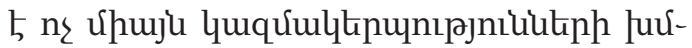

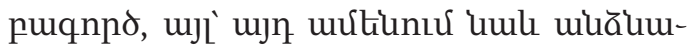

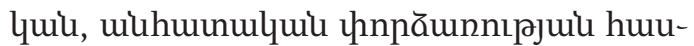
quigưurip $\mathrm{l}$, puin 5 nıрjuil, qhunulyuil huulugnıpjnilitiknıu uנn huulugluonıpjuiu utplyujugúurip pupkingnn ưn-

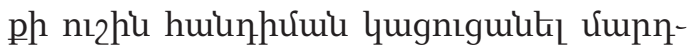

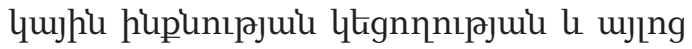

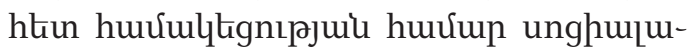

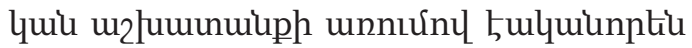

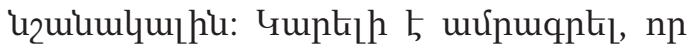

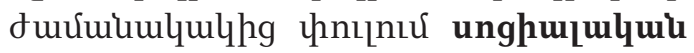

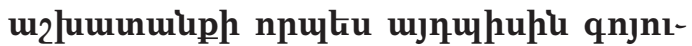
pjnilu utiplujuinnú 5 npultu tnuúpuulunıpjnili qhunnıpjnil, úwuluwqhunuquil upltiunulnp qnponititnıpjnil b nıunıgulyupq:

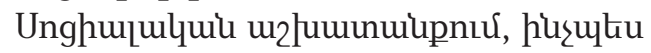

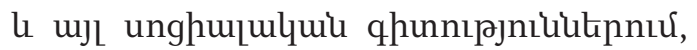
uiuhpudtizunntiu unuquiunu 5 ungh-

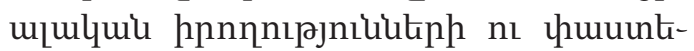
nh huulqugúuil no vitlunıpjuil buinhp:

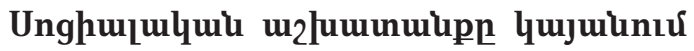

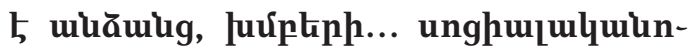

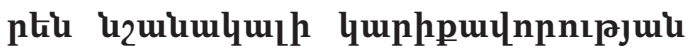
huulugúuúp nt vitlunqupup pugn-

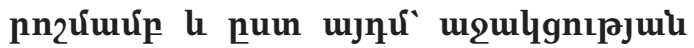
hpuqnporuúp: bl bulqui unuiăhuhuunlnıpjnila 5 uwa uju, np unghuцu-

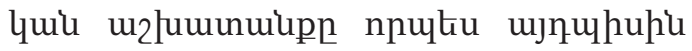

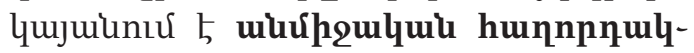
gnıpjurip: Unghu[ulqui w2 wumunuiph

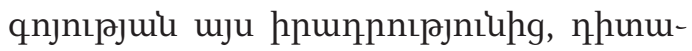

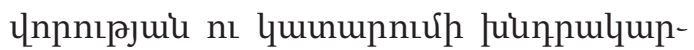
qhg lupkilh 5 ulquunkl, np huulqugúuiu,

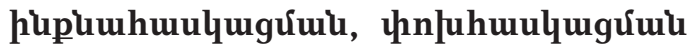
no vitilumpulinıpjui julppulqupqn ulqqpniupnptiu utiphjnıuц wo b unghuцш- 


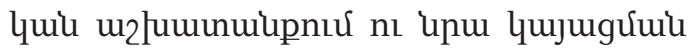

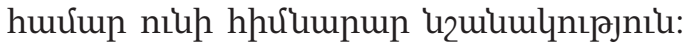

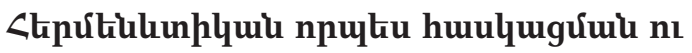

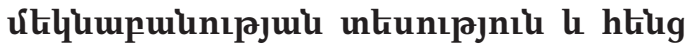
uthluuqhunnıpjnilu, hnıưulhunup le ungh-

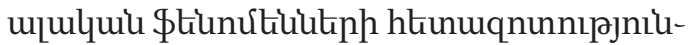

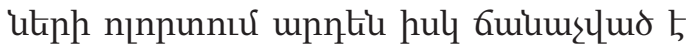

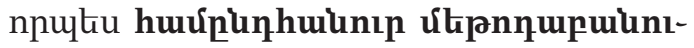

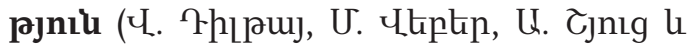

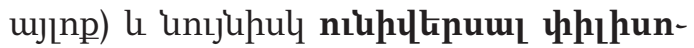
hujulyul hujtigulupq ( $<.-9$ 9unu-

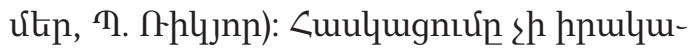

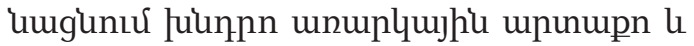

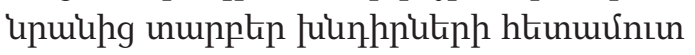
ununkgnıu, np huunnıl 5 ulyuunцnıu hipu

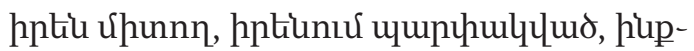

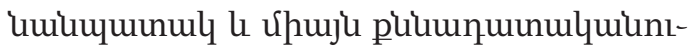

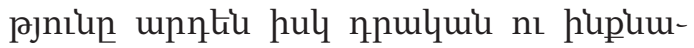

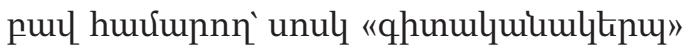

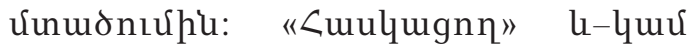

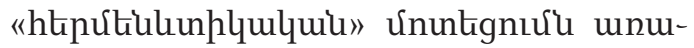

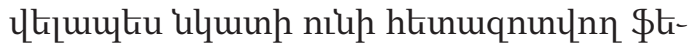

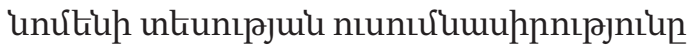

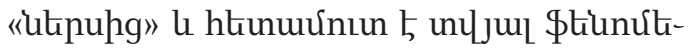
up "hulnıpjulu purgnnn2nıú 'utplujugnn untunıрjuil utepph hungunpnuर्utu-

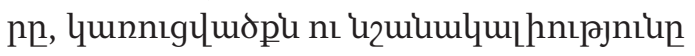
puguhujunt]nı nhunuцnnnıрjuil lı puin

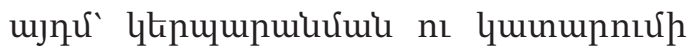
juinpulyupqhu:

Unghujulqui ur tumumuph \$tiun-

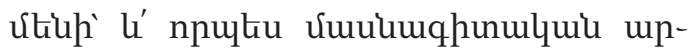
lthuonulnp qnponithnıpjuiu intuulyh,

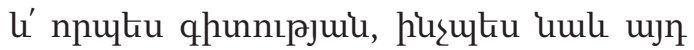
nцnpunpiu ltepuptipnn untunıpjniutuph

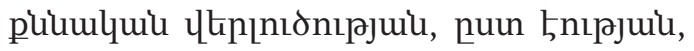
uluhujun, pujg l. huuwitilh npnitihp

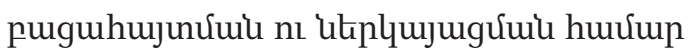

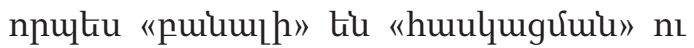

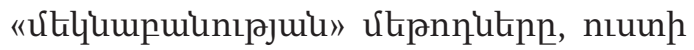

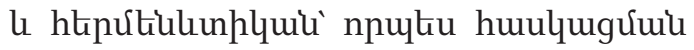

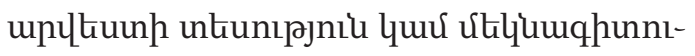
pjniu: Unghuцulyuiu hłunuqnunnıpjnil- ukph nцnpunnư nten XX ๆ. ulqphg uluu-

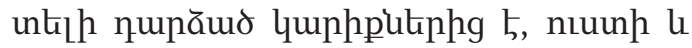
ujn quiphph intuuilynituhg insjuutu

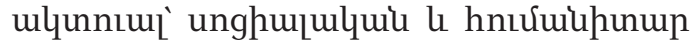
qhunnıpjniuutph hứpiunhuiunıp ukpn-

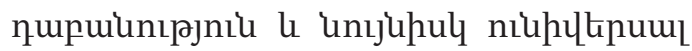
hhlhunhujulquil hujtgulyunq Guiuusunn htinutulumplqujh lnulptiun lhpunnepjnikn, npi hpulquiuugunıর

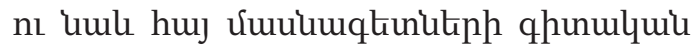
w2luwunnıpjniututpniv wjuupup ungh-

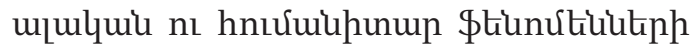

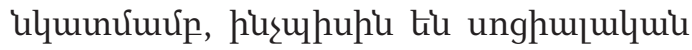
urluuunuiupp, unghnpnqhuil l pupkqnp-

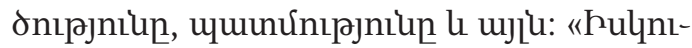

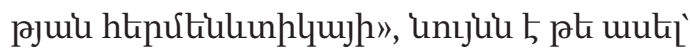
hulnıрjuil puguhujunরuin hłunuर्unıun uthluwqhunnıرuiu, hujtgulqunqny hpuquiuugluo htunuqnunnıpnituteplu hptiug

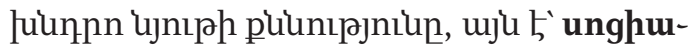

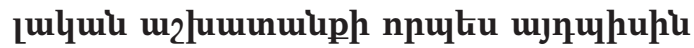
qnjnıpjuil hulnıpjuil pugnpn2nıúp,

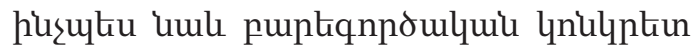

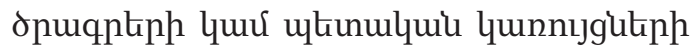
qnponiunıрjuiu nı пpuiugnıर ujl uuje hp

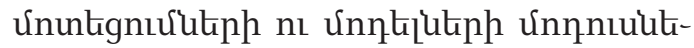
pny hpuqnpolud «unghujulyuil u2luu-

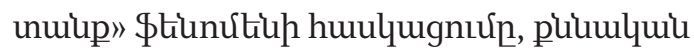

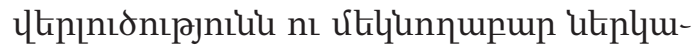

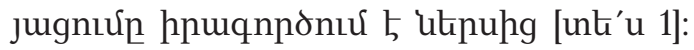

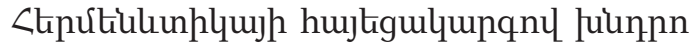
ujnıр nıunıর'uuuppnıрjnilu hpulyuiuug-

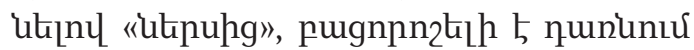
nıunıưuuupplnn \$tiunútiuteph qnjnıpjuiu hulnıpjniun le huupulnnnıpjnil

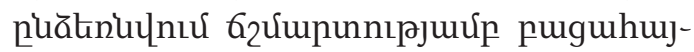

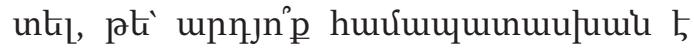
unghujulyuil w2 kuwumulph nı puptiqnp১ulquil dpuqph qnponilitnıрjuiu ununtil-

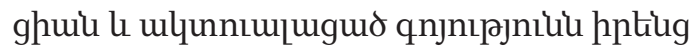

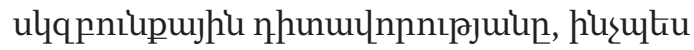

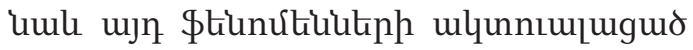
qnjnıpjniun' hpting ununtighujhì: 


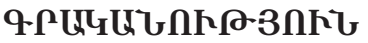

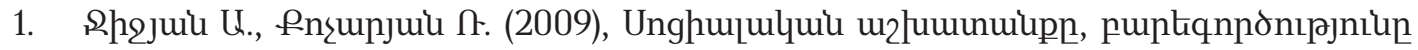

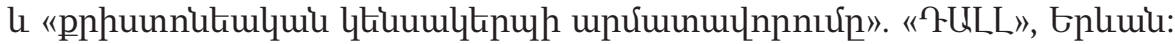

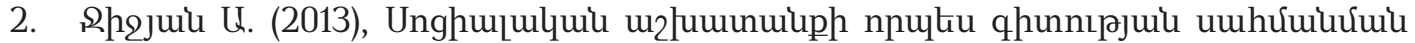

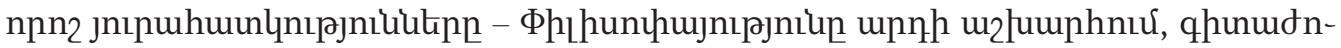

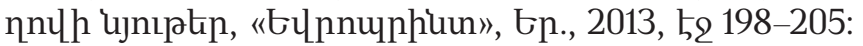

3. Муздыбаев К. Переживание бедности как социальной неудачи: атрибуция ответственности, стратегии совладения и индикаторы депривации. http://www. nir.ru/sj/sj/sji-a/muz .html

4. Погосян Г. А., Современное армянское общество: особенности трансформации. М.: «Академия», 2005.

5. Социальная работа, под ред. В. И. Курбатова. «Феникс», Ростов на Дону, 2000.

6. Healy L. M., (2001), International social work. Oxford University Press.

7. McDonald C. (2006), Challenging Social Work. The institutional context of practice. Palgrave Macmillan, London.

8. Morales A. T. \& Sheafor B. W. (1992), Social work. A profession of many faces., Boston.

\section{SOCIAL WORK IN TRANSFORMING SOCIETY}

\section{ROMIK KOCHARYAN}

«Matenadaran», Senior Researcher,P hD in Philosophical Sciences Assistant of the Chair of Sociology and Social work, ASPU after Kh. Abovyan

\section{ANAHIT JIJYAN}

ASPU after Kh. Abovyan, Chair of Sociology and Social Work, Assistant, Ph. D

The paper are reveals the specificities of formation and presence of social work in the "transforming society" of the Republic of Armenia. In contemporary Armenia the existence of the social work as such is present in three modes as professional activity, as educational discipline and also as a science.

\section{СОЦИАЛЬНАЯ РАБОТА В ТРАНСФОРМАЦИОННОМ ОБЩЕСТВЕ \\ РОМИК КОЧАРЯН \\ «Матенадаран», ст. науч. сотр., к.ф.н. ассистент кафедры социологии и социальной работы АГПУ им. Х. Абовяна}

\section{АНАИТ ЖИДЖЯН}

АГПУ им. Х. Абовяна, кафедра сощиологии и социальной работы, ассистент, к. ф.н

В данной статье выявлены особенности формирования и присутствия социальной работы в «трансформационном обществе» РА. В современной Армении социальная работа присутствует в трёх ипостасях: как профессиональная деятельность, как обучающая дисциплина и как наука. 